\title{
ONE-STEP VITRIFICATION OF MURINE EMBRYOS CHALLENGES CURRENT PARADIGMS OF CRYOBIOLOGY
}

Fabien Ectors ${ }^{1,2 *}$, Pierre Vanderzwalmen ${ }^{1,3,4}$, Nadine Dupuis ${ }^{1}$, Delphine Connan ${ }^{1} \&$ Luc Grobet $^{1,3}$

${ }^{1}$ VitriCell S.A., Liège, Belgium; ${ }^{2}$ GIGA Mouse Transgenics Facility, Liège, Belgium; ${ }^{3}$ Embryology Unit, Morphology \& Pathology Department, Faculty of Veterinary Medicine, University of Liège, Liège, Belgium; ${ }^{4} \mathrm{ZECH}$ IVF Centers, Bregenz, Austria \& CHIREC, Braine l'Alleud, Belgium.

\section{*Corresponding Author: Fabien.Ectors@vitricell.com}

Cryopreservation of embryos is amongst the most powerful tools for preserving the genetics of laboratory animals. Vitrification is widely recognized as more efficient than slow freezing e.g. in human assisted reproduction technologies and for preserving murine embryos. Unfortunately, current vitrification procedures require multiple pre-cooling and post-warming exposure steps to dedicated solutions to reach maximum effectiveness, which appears difficult to deal with when many embryos are cryopreserved in one single session. To help solving this issue, we have developed a one-step embryo vitrification procedure. Briefly, embryos are exposed to a unique -chemically defined-vitrification solution before plunging in the liquid nitrogen. Subsequent warming is performed by immersing the vitrified embryos directly into the culture medium.

Murine embryos at the zygote, two cells and morula stages have undergone our one-step procedure either under aseptic or non-aseptic conditions. After warming, direct in vitro survival, development to the blastocyst stage and in vivo development to birth were recorded as endpoints. Short exposure times to the vitrification solution (less than 60 seconds) before cooling and direct warming into culture medium led to results equivalent or better than after classical vitrification. Longer exposure times to the vitrification solution (between 90 and 150 seconds) decreased efficiency.

These results demonstrate that intracellular vitrification after our one-step procedure occurs by combined effects of fast cooling (or warming) and cell dehydration, with minimal, if any, ingress of cryoprotectants. The absence of deleterious effect of warming directly into the culture medium and the relatively low sensitivity to thermal inertia (aseptic vs non-aseptic) of the carrier is a confirmation thereof. 
Consequently, beyond bringing a methodological simplification without any loss of efficiency, our patented one-step vitrification procedure dramatically lowers if not suppresses intracellular concentration of cryoprotectants and associated toxicity, thereby challenging some commonly accepted concepts of cryobiology.

Source of funding: This study was jointly funded by the University of Liège, the GIGA Research Center, and the Walloon Region-DGO6 (FSO grant $\mathrm{N}^{\circ} 1217842$ ).

Conflict of interest: None to disclose. 